\title{
Local preferential attachment model for hierarchical networks
}

\author{
Li-Na Wang ${ }^{\text {a,b }}$, Jin-Li Guo ${ }^{\mathrm{a}, *}$, Han-Xin Yang ${ }^{\mathrm{c}}$, Tao Zhou ${ }^{\mathrm{c}, \mathrm{d}}$ \\ ${ }^{a}$ Business School, University of Shanghai for Science and Technology, Shanghai 200093, PR China \\ ${ }^{\mathrm{b}}$ College of Sciences, Inner Mongolian University of Technology, Hohhot 010051, PR China \\ ${ }^{\mathrm{c}}$ Department of Modern Physics, University of Science and Technology of China, Hefei 230026, PR China \\ ${ }^{\mathrm{d}}$ Department of Physics, University of Fribourg, Fribourg 1700, Switzerland
}

\begin{abstract}
In real-life networks, incomers may only connect to a few others in a local area for their limited information, and individuals in a local area are likely to have close relations. Accordingly, we propose a local preferential attachment model. Here, a local-area-network stands for a node and all its neighbors, and the new nodes perform nonlinear preferential attachment, $\pi\left(k_{i}\right) \propto k_{i}^{\alpha}$, in local areas. The stable degree distribution and clusteringdegree correlations are analytically obtained. With the increasing of $\alpha$, the clustering coefficient increases, while assortativity decreases from positive to negative. In addition, by adjusting the parameter $\alpha$, the model can generate different kinds of degree distribution, from exponential to power-law. The hierarchical organization, independent of $\alpha$, is the most significant character of this model.
\end{abstract}

\section{Introduction}

In the past few years, arrays of discoveries have been added to our understanding of complex networks. With the improvement of computing power, it is possible to study the statistical properties of huge size networks. Some interesting results are found such as large clustering coefficients and power-law degree distributions. Fitting to the small-world phenomena in real world, Watts-Strogatz model [1] with large clustering coefficient and short average distance was presented. To capture the 'rich gets richer' phenomenon, Barabási and Albert proposed a scale-free model (BA model) [2], which generates a network with a power-law degree distribution. It is found that growth and preferential attachment are essential to the emergence of a scale-free structure [3]. Though the BA model captures those fundamental mechanisms, it only predicts a fixed exponent, while real networks display various exponents. Therefore, many scientists [4-13] have proposed some extensions to make the models more realistic.

For some large-scale complex systems, a new individual entering the system will consume a great deal of searching costs when doing the global preferential attachments. It has been observed that the preferential attachment within local economy regions exists in the World Trade Web [14]. A similar phenomenon is in the Internet on the router level where a host, mostly, only has information of other hosts in a local domain [15,16]. Based on above considerations, Li and Chen [17] presented a Local World model, in which $M$ randomly selected nodes constitutes a local-world wherein a new node does local preferential attachments. To modify the small clustering property in the Local World model [17], Zhang et al. [18] introduced an evolving scale-free network model with a continuously adjustable clustering coefficient. Chen et al. [19] proposed a multi-local-world model to mimic the Internet. Wu and Liu [20] proposed a community network model with a high clustering coefficient. In previous studies, the local world is composed of randomly selected nodes, thus a node in a local

\footnotetext{
* Corresponding author.

E-mail addresses: phd5816@163.com (J.-L. Guo), zhutou@ustc.edu (T. Zhou).
} 


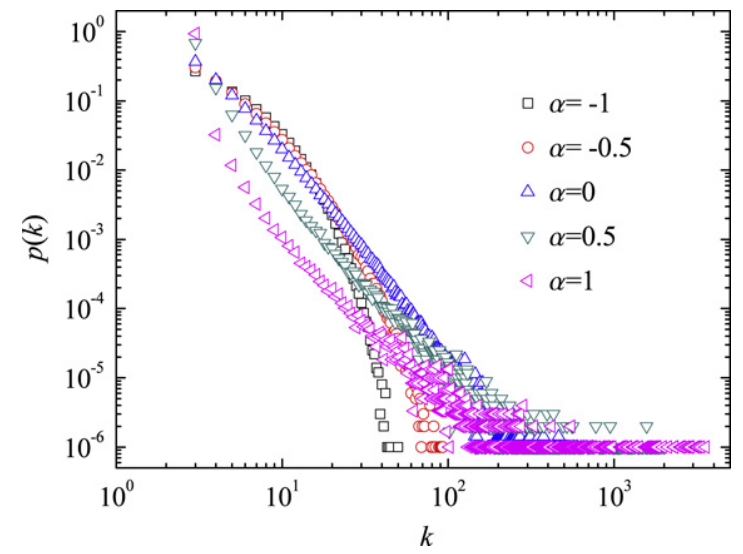

Fig. 1. (Color online) The degree distributions for different $\alpha$ in a log-log plot with $m=m_{0}=3$. Here $p(k)$ denotes the probability density of degree $k$. Each data point is obtained by averaging over 20 independent realizations with network size $N=10^{6}$ fixed.

world may not have denser connections compared with its connections to the nodes outside this local world. However, in real-life networks, individuals in a local area are likely to have closer relations. For example, the routers have, generally, much denser connections within a local domain than those between different domains. The observed community structure [21] has just embodied this concept as well. Accordingly, we present a local preferential attachment model where a local area consists of a node and all its neighbors. To be distinguishable, we call a node and all its neighbors a local-area-network (LAN) instead of local world. The newly added nodes perform nonlinear preferential attachments, as $\pi\left(k_{i}\right) \propto k_{i}^{\alpha}$, in the LANs, where $k_{i}$ is the degree of the ith node and $\alpha$ is a free parameter (Note that, this nonlinear preferential function is wellknown as a global attachment mechanism [22-25]). The hierarchical organization, independent of $\alpha$, is the most significant character of this model.

The rest of this paper is organized as follows: Section 2 introduces the model, followed by simulation results and analytical calculations for degree distribution in Section 3 and clustering-degree correlation in Section 4. Finally, we summarize our work in Section 5. The simplest case where each new node only attahches one edge in the LAN, is discussed in the Appendix A.

\section{Local preferential attachments model}

A new node entering the network is denoted by an event $I$. Based on the spirit of the customer input process in a random service system [26], it is supposed that the coming processing of nodes can be well described by a Poisson process with parameter $\lambda$ which denotes the mean number of the occurrences of $I$ in a unit time. That is to say, when $\lambda=1$, there is on average one new node entering into the network in a unit time. The model is as follows:

(1) Random growth: network starts with $m_{0}$ fully connected nodes. At each time step, a new node with $m\left(\leq m_{0}\right)$ edges, is added to the network. Here, the nodes come obeying a Poisson process with parameter $\lambda=1$, and an LAN is defined as a randomly selected node and all its neighbors. For a given LAN, we call the central node the root node.

(2) Local preferential attachments: the newly added node is connecting to a node $i$ in an LAN with probability

$$
\pi\left(k_{i}\right)=\frac{k_{i}^{\alpha}}{\sum_{j \in L} k_{j}^{\alpha}},
$$

where $L$ denotes the set of all nodes in this LAN, and the sum goes over all the nodes belonging to $L$. Note that, for each newly added node, the $m$ times of selections of different root nodes (thus different LANs) are completely independent.

After $t$ time steps, this procedure results in a network with $N=m_{0}+t$ nodes and $E=m t+E_{0}$ edges, where $E_{0}=\frac{m_{0}\left(m_{0}-1\right)}{2}$ is the number of edges in the initial network. The mean node degree is

$$
\bar{k}=\frac{2 E}{N}=\frac{2 m t+2 E_{0}}{m_{0}+t} \rightarrow 2 m(t \rightarrow \infty) .
$$

\section{Degree distribution}

As shown in Fig. 1, by adjusting the parameter $\alpha$, the model can generate different kinds of degree distribution, from exponential to power-law.

An existing node $i$, before the $(t+1)$ th event occurs, can be in one of three situations: (i) being selected as the root node with probability $\frac{1}{m_{0}+t}$; (ii) belonging to the LAN but not being the root with probability $\frac{k_{i}}{m_{0}+t}$; (iii) outside the LAN with probability $1-\frac{k_{i}+1}{m_{0}+t}$. Therefore, we get the changing rate of $i$ 's degree, as: 


$$
\frac{\partial k_{i}}{\partial t}=\frac{1}{m_{0}+t} \cdot m \cdot \frac{k_{i}^{\alpha}}{\sum_{j \in L_{i}} k_{j}^{\alpha}}+\frac{k_{i}}{m_{0}+t} \cdot m \cdot \frac{k_{i}^{\alpha}}{\sum_{j \in L_{s}} k_{j}^{\alpha}} ; \quad k_{i}\left(t_{i}\right)=m,
$$

where $t_{i}$ denotes the time that the $i$ th node enters the network, $k_{i}(t)$ denotes the degree of node $i$ at time $t, L_{i}$ denotes the set of nodes in the LAN with root node $i$ and $L_{S}$ represents an LAN containing $i$ where the root $s$ is a neighbor of $i$. In Eq. (3), the first term accounts for the situation (i), while the second term accounts for the situation (ii). The formulae reported here are very similar to those in Refs. [12,24].

\subsection{The case $\alpha=0$}

From Eq. (3), neglecting the effect of degree-degree correlation, a zero-order approximation can be obtained as:

$$
\sum_{j \in L_{i}} k_{j}^{\alpha}=\sum_{j \in L_{s}} k_{j}^{\alpha} \stackrel{\text { def }}{=} \sum_{j \in L} k_{j}^{\alpha}=\bar{k}^{\alpha} \cdot \bar{k}+k_{i}^{\alpha},
$$

where $L$ denotes the set of nodes in the LAN excluding the root. Note that, this approximation works well only if the assortativity is close to zero (see below the definition and simulation on assortativity), thus for large positive $\alpha$, the approximation is rough. Substituting Eq. (4) into Eq. (3), we can get

$$
\frac{\partial k_{i}}{\partial t}=\frac{k_{i}+1}{m_{0}+t} \cdot \frac{m k_{i}^{\alpha}}{\sum_{j \in L} k_{j}^{\alpha}}=\frac{k_{i}+1}{m_{0}+t} \cdot \frac{m k_{i}^{\alpha}}{\overline{k^{\alpha} \cdot \bar{k}+k_{i}^{\alpha}}} ; \quad k_{i}\left(t_{i}\right)=m .
$$

Next, we provide the analytical solution for two special cases: $\alpha=0$ (analysis of the simplest case with $\alpha=0$ and $m=1$ is presented in the Appendix $\mathrm{A}$ ) and $\alpha=-1$.

When $\alpha=0$, one gets

$$
\frac{\partial k_{i}}{\partial t}=\frac{m}{m_{0}+t} \cdot \frac{k_{i}+1}{\frac{2 m t+2 E_{0}}{m_{0}+t}+1} \approx \frac{m}{2 m+1} \cdot \frac{k_{i}+1}{t} .
$$

Solving this differential equation, one obtains

$$
k_{i}(t)=(m+1)\left(\frac{t}{t_{i}}\right)^{\frac{m}{2 m+1}}-1,
$$

therefore, one has

$$
P\left\{k_{i}(t)<k\right\}=P\left\{t_{i}>g(k, t)\right\},
$$

where

$$
g(k, t)=t \cdot\left(\frac{m+1}{k+1}\right)^{2+\frac{1}{m}} .
$$

Since the nodes come in a Poisson process with parameter $\lambda=1$, the entering time $t_{i}$ obeys an $\Gamma$-distribution [26]. Therefore, Eq. (8) can be rewritten as

$$
P\left\{k_{i}(t)<k\right\}=1-P\left\{t_{i} \leq g(k, t)\right\}=\mathrm{e}^{-g(k, t)} \sum_{l=0}^{i-1} \frac{(g(k, t))^{l}}{l !},
$$

and

$$
P\left\{k_{i}(t)=k\right\}=\frac{\partial P\left\{k_{i}(t)<k\right\}}{\partial k}=-\mathrm{e}^{-g(k, t)} \frac{\partial g(k, t)}{\partial k} \frac{(g(k, t))^{i-1}}{(i-1) !} .
$$

Thus the stable degree distribution reads

$$
\begin{aligned}
p(k) & =\lim _{t \rightarrow \infty} \frac{1}{t} \sum_{i=1}^{t} P\left\{k_{i}(t)=k\right\} \approx \lim _{t \rightarrow \infty} \frac{1}{t} \sum_{i=1}^{\infty} P\left\{k_{i}(t)=k\right\}=-\lim _{t \rightarrow \infty} \frac{1}{t} \frac{\partial g(k, t)}{\partial k} \\
& =\left(2+\frac{1}{m}\right) \cdot(m+1)^{2+\frac{1}{m}} \cdot(k+1)^{-\left(3+\frac{1}{m}\right)} .
\end{aligned}
$$

It is a power-law distribution for large $k$, which means a scale-free network can be obtained via random attachments in LANs, with power-law exponent $\gamma=3+\frac{1}{m}$. 

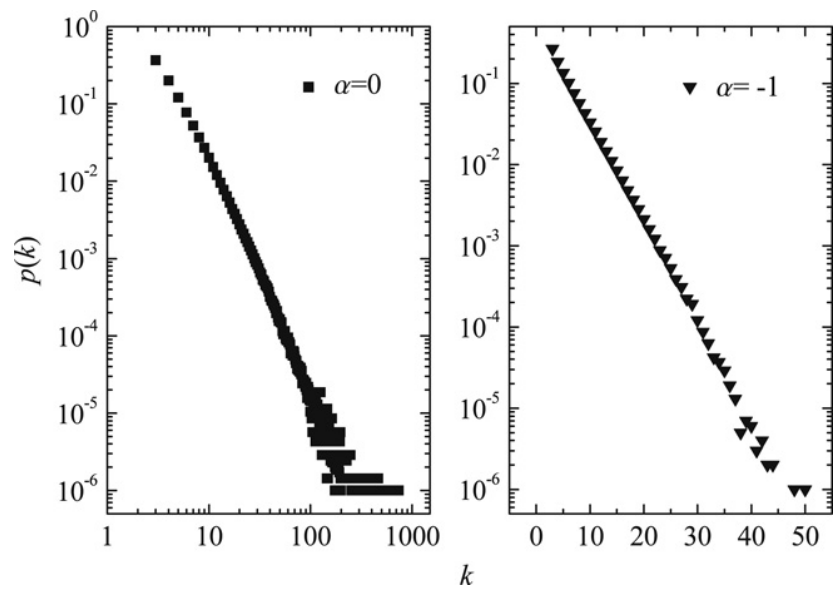

Fig. 2. Degree distributions for $\alpha=0$ in a log-log plot and $\alpha=-1$ in a linear-log plot with $m=m_{0}=3$. Each data point is obtained by averaging over 20 independent realizations with network size $N=10^{6}$ fixed.

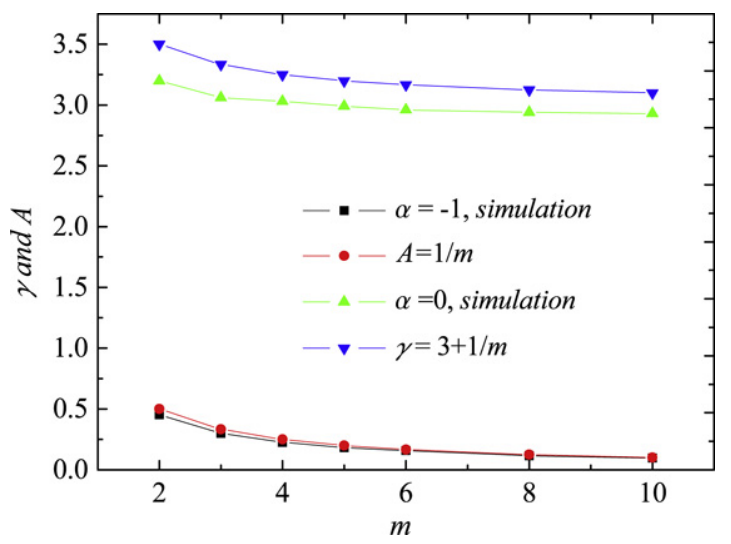

Fig. 3. (Color online): Degree distributions for different $m$. Each numerical point is obtained by averaging over 20 independent realizations with network size $N=10^{6}$ fixed. The simulation agrees well with analysis for large $m$.

3.2. The case $\alpha=-1$

When $\alpha=-1$, we can obtain

$$
\frac{\partial k_{i}}{\partial t}=\frac{m}{m_{0}+t} \cdot \frac{\left(k_{i}+1\right) k_{i}^{-1}}{1+k_{i}^{-1}} \approx \frac{m}{t} .
$$

Solving this differential equation, one gets

$$
k_{i}(t)=m \ln \frac{t}{t_{i}}+m
$$

Using a similar method to that as used above, one can get

$$
p(k)=\frac{e}{m} \cdot \mathrm{e}^{-\frac{k}{m}} \propto D \mathrm{e}^{-A k},
$$

where $D$ is the normalization constant and $A=\frac{1}{m}$. Clearly, it is an exponential distribution for large $k$.

Fig. 2 reports the simulation results on degree distributions of the two specific cases: $\alpha=0$ and $\alpha=-1$. The former obeys a power-law form and the latter follows an exponential form, which is qualitatively in accordance with our analytical results. Furthermore, in Fig. 3, we compared the quantitative predictions of exponents in Eq. (12) and Eq. (15) with the simulation results. We have not obtained the analytical expression for arbitrary $\alpha$. The only clear point is that the degree distribution will become broader, namely the network will become more heterogeneous, with increasing $\alpha$. 

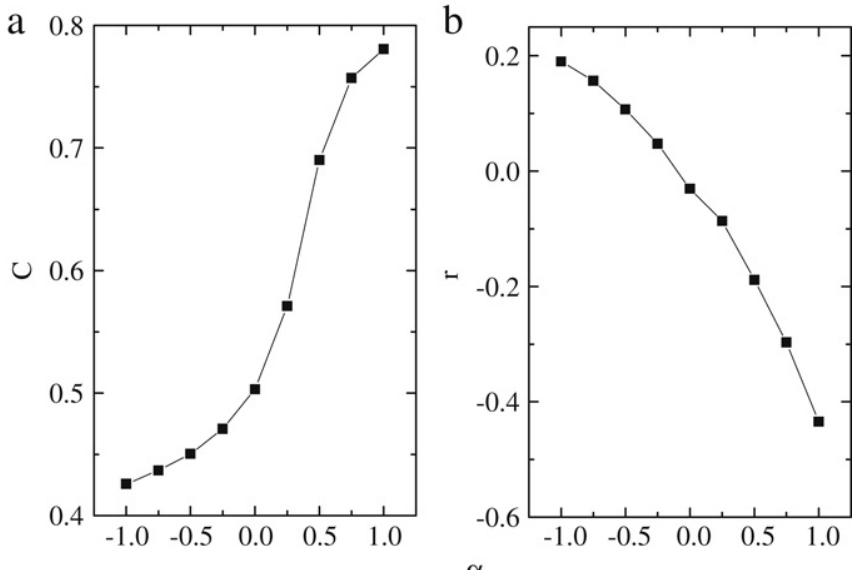

Fig. 4. Clustering coefficient (a) and assortative coefficient (b) as functions of $\alpha$. Each data point is obtained by averaging over 20 independent realizations with network size $N=10^{6}$ and $m=m_{0}=3$ fixed.



a

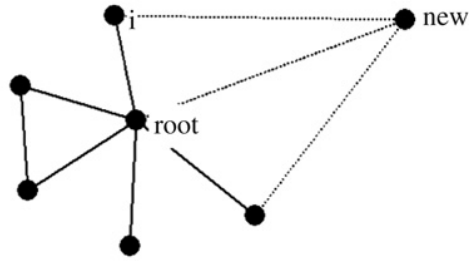

$\mathrm{b}$

Fig. 5. Illustration of the changes of $e_{i}$. In situation (a), node $i$ is the root of the LAN, while in situation (b), node $i$ is in the LAN but not the root.

\section{Clustering-degree correlations}

High clustering is a typical property of social acquaintance networks, where two individuals with a common friend are likely to know each other. Given an arbitrary node $i$, the most immediate measure of clustering is the clustering coefficient $C_{i}$, which is the ratio between the number of acutal existing edges $e_{i}$ and the number of all possible edges $k_{i}\left(k_{i}-1\right) / 2$ among node $i$ 's neighbors, and the clustering coefficient $C$ of the whole network is $C=\frac{1}{N} \sum_{i} C_{i}$. As shown in Fig. 4(a), $C$ increases with increasing $\alpha$.

The clustering-degree correlation $C(k)$ denotes the average clustering coefficient of nodes with degree $k$. In many real networks, the clustering scales as $C(k) \propto k^{-1}$, which represents the hierarchical organization of structure [27]. To calculate the clustering-degree correlations in each time step, we need to consider the changing rate of $e_{i}$ for an arbitrary node $i$. Denoting $L$ as the set of nodes in the LAN, there are two cases where $e_{i}$ will increase:

(a) Node $i$ is the root node of an LAN and connected to the new node, whose probability is $\frac{1}{m_{0}+t} \cdot \frac{m k_{i}^{\alpha}}{\sum_{j \in L} k_{j}^{\alpha}}$ and $e_{i}$ increases by $m-1(m>1)$ (see Fig. 5(a)).

(b) One neighbor of node $i$ is the root of an LAN, that is to say $i \in L$ as well. Supposing the number of node $i$ 's neighbors in this LAN is $n(n \geq 1)$, and node $i$ itself as well as $h(1 \leq h \leq l) i$ 's neighbors are connected to the new node. The probability of this situation is

$$
\sum_{h=1}^{l}\left[\frac{k_{i}}{m_{0}+t} \cdot C_{m}^{h+1} \cdot\left(\frac{k_{i}^{\alpha}}{\sum_{j \in L} k_{j}^{\alpha}}\right) \cdot\left(\frac{\bar{k}^{\alpha}}{\sum_{j \in L} k_{j}^{\alpha}}\right)^{h}\right],
$$

and $e_{i}$ increases by $h$, where $l=\min (m-1, n), m>1$. Neglecting high order terms $(h>1)$, we get an approximation as $\frac{1}{2} \cdot \frac{k_{i}}{m_{0}+t} \cdot \frac{m k_{i}^{\alpha}}{\sum_{j \in L} k_{j}^{\alpha}} \cdot \frac{(m-1) \bar{k}^{\alpha}}{\sum_{j \in L} k_{j}^{\alpha}}$, where $e_{i}$ increases by 1 (See Fig. 5(b)).

Based on the above analysis, we can obtain the changing rate of $e_{i}$ :

$$
\frac{\partial e_{i}}{\partial t}=\frac{1}{m_{0}+t} \cdot \frac{m(m-1) k_{i}^{\alpha}}{\sum_{j \in L} k_{j}^{\alpha}}+\frac{1}{2} \cdot \frac{k_{i}}{m_{0}+t} \cdot \frac{m k_{i}^{\alpha}}{\sum_{j \in L} k_{j}^{\alpha}} \cdot \frac{(m-1) \bar{k}^{\alpha}}{\sum_{j \in L} k_{j}^{\alpha}},
$$




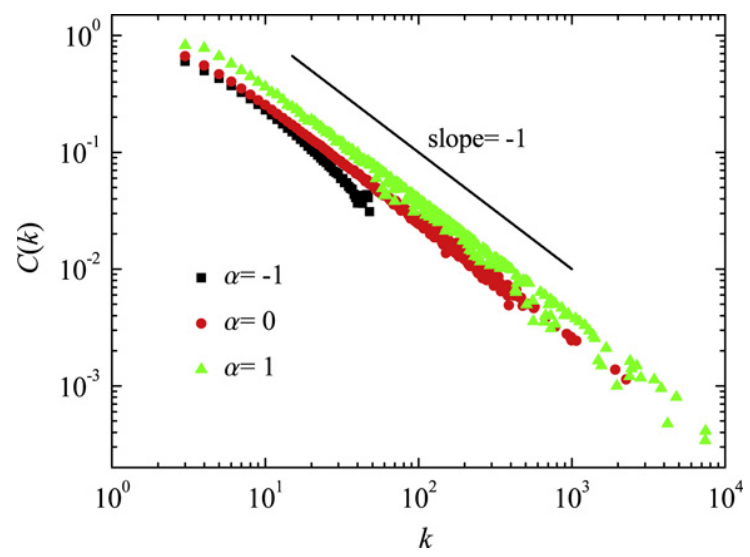

Fig. 6. (Color online) The clustering coefficient as a function of the node degree for different $\alpha$ in a $\log$-log plot with $m=m_{0}=3$. Each data point is obtained by averaging over 20 independent realizations with network size $N=10^{6}$ fixed. The straight line of slope -1 is plotted as a guide to eyes.

and substituting Eq. (5) into Eq. (16), one obtains

$$
\begin{aligned}
\frac{\partial e_{i}}{\partial t} & =\frac{m-1}{k_{i}+1} \cdot \frac{\partial k_{i}}{\partial t}+\frac{m-1}{2} \cdot \frac{k_{i}}{k_{i}+1} \cdot \frac{1}{\frac{2 m t+2 E_{0}}{m_{0}+t}+1} \cdot \frac{\partial k_{i}}{\partial t} \\
& \approx \frac{m-1}{k_{i}+1} \cdot \frac{\partial k_{i}}{\partial t}+\frac{m-1}{4 m+2} \cdot \frac{k_{i}}{k_{i}+1} \cdot \frac{\partial k_{i}}{\partial t} .
\end{aligned}
$$

The solution of Eq. (17) leads to:

$$
e_{i}=e_{i, 0}+\frac{m-1}{4 m+2} k_{i}(t)+\frac{(4 m+1)(m-1)}{4 m+2} \ln \left(k_{i}(t)+1\right) .
$$

Ignoring the first term in Eq. (18), the clustering scales, independent of parameter $\alpha$, as $C(k) \propto k^{-1}$ for large $k$.

$$
C(k)=\frac{2 e}{k(k-1)} \approx \frac{m-1}{2 m+1} \cdot \frac{1}{k}+\frac{(4 m+1) \cdot(m-1)}{2 m+1} \cdot \frac{\ln k}{k^{2}} \propto \frac{1}{k} .
$$

This nontrivial correlation between clustering coefficient and degree, as the characteristic of hierarchical structure, is observed in many real-life and artificial networks [27-30]. Fig. 6 shows the numerical simulations, which agree well with the analytical results.

\section{Conclusion}

Considering an individual's limited ability and the interrelationship in a local area, in this paper, we proposed a local preferential attachment model. Based on a theory of the Poisson process, the stable degree distributions of the network and clustering-degree correlations are analytically obtained. By adjusting the parameter $\alpha$, the model can generate different kinds of degree distribution, from exponential to power-law. And with $\alpha$ 's increasing from -1 to 1 , the clustering coefficient increases while the assortative coefficient decreases from positive to negative (see Fig. 4(b)). The hierarchical organization, independent of parameter $\alpha$, is the most significant property of this model.

\section{Acknowledgements}

This work was supported by the National Natural Science Foundation of China under Grant No. 70871082 and the Foundation of Shanghai Leading Academic Discipline Project (Grant Nos. T0502, S30504). T. Z. acknowledges the National Natural Science Foundation of China under Grant No. 10635040.

\section{Appendix. The simplest case with $\alpha=0, m=1$}

New nodes linked with only one edge will create a tree graph. Next we provide the analytical solution of degree distribution when $m=1, \alpha=0$. Considering the probability that any two nodes connect in the network, in Eq. (3), one has

$$
\left\{\begin{array}{l}
\sum_{j \in L_{i}} k_{j}^{\alpha}=\sum_{j \in L_{i}} 1=k_{i}+1 \\
\sum_{j \in L_{s}} k_{j}^{\alpha}=q_{i s} \cdot\left(k_{s}+1\right) \approx q(\bar{k}+1)=\frac{3 t+2 E_{0}+m_{0}}{t+m_{0}} \cdot q,
\end{array}\right.
$$




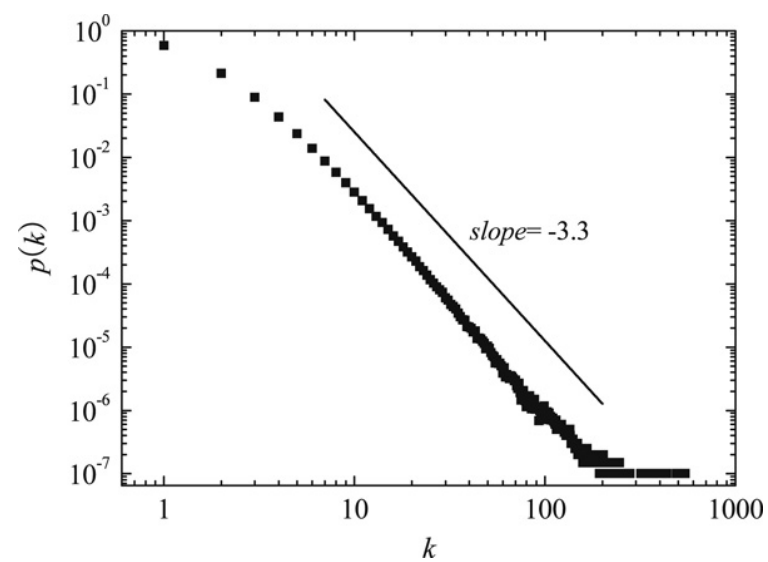

Fig. 7. Degree distributions for $m=1, \alpha=0$. The power-law degree distribution exponent is 3.3. Each data point is obtained by averaging over 20 independent realizations with network size $N=10^{7}$ fixed.

where $q_{i s}$ denotes the probability that node $s$ is a neighbor of node $i$, and $q \in(0,1)$ denotes the mean probability that any two nodes connect in the network. For a fixed $\alpha$ and $m, q$ is a constant parameter. Then the differential equation changes

$$
\frac{\partial k_{i}}{\partial t} \approx \frac{1}{t} \cdot\left(\frac{1}{k_{i}+1}+\frac{k_{i}}{3 q}\right) ; \quad k_{i}\left(t_{i}\right)=1
$$

For a large $t$, neglecting the first term on the right side in the above equation, then solving Eq. (21), one obtains

$$
k_{i}(t)=\left(\frac{t}{t_{i}}\right)^{\frac{1}{3 q}}
$$

Since the nodes come in a Poisson process with parameter $\lambda=1$, the entering time $t_{i}$ obeys an $\Gamma$-distribution [26]. Therefore, one gets

$$
P\left\{k_{i}(t)<k\right\}=P\left\{t_{i}>t k^{-3 q}\right\}=1-P\left\{t_{i} \leq t k^{-3 q}\right\}=\mathrm{e}^{-t k^{-3 q}} \sum_{l=0}^{i-1} \frac{\left(t k^{-3 q}\right)^{l}}{l !},
$$

and

$$
P\left\{k_{i}(t)=k\right\}=\frac{\partial P\left\{k_{i}(t)<k\right\}}{\partial k}=-\mathrm{e}^{-t k^{-3 q}} \cdot \frac{\partial\left(t k^{-3 q}\right)}{\partial k} \cdot \frac{\left(t k^{-3 q}\right)^{i-1}}{(i-1) !} .
$$

Then the stable degree distribution, when $m=1, \alpha=0$, reads

$$
\begin{aligned}
p(k) & =\lim _{t \rightarrow \infty} \frac{1}{t} \sum_{i=1}^{t} P\left\{k_{i}(t)=k\right\} \approx \lim _{t \rightarrow \infty} \frac{1}{t} \sum_{i=1}^{\infty} P\left\{k_{i}(t)=k\right\}=-\lim _{t \rightarrow \infty} \frac{1}{t} \cdot \frac{\partial\left(t k^{-3 q}\right)}{\partial k} \\
& =3 q k^{-(3 q+1)} .
\end{aligned}
$$

It is a power-law distribution with exponent $3 q+1(\in(1,4))$, which means it is a scale-free tree graph when a new node, linked with only one edge, is randomly attached in the LAN. Here, the power-law exponent is relevant to parameter $q$, the value of which we give as follows. The mean value of degree $k$ can be got from $\bar{k}=\int_{k_{\min }}^{k_{\max }} k p(k) \mathrm{d} k$, where $k_{\min }$ and $k_{\max }$ are the minimum and maximum node degree in the network respectively. Therefore, when the network scale is sufficiently large, namely $t \rightarrow \infty(m=1, \alpha=0)$, one gets approximately

$$
\int_{1}^{\infty} k p(k) \mathrm{d} k=2 .
$$

Substituting $p(k)=3 q k^{-(3 q+1)}$ into Eq. (26), $q=\frac{2}{3}$ is obtained. Thus the theoretical value of the power-law exponent is $\gamma=3$. The Fig. 7 reports the simulation results on degree distributions. 


\section{References}

[1] D.J. Watts, S.H. Strogatz, Nature 393 (1998) 440

[2] A.-L. Barabási, R. Albert, Science 286 (1999) 509.

[3] A.-L. Barabási, R. Albert, H. Jeong, Physica A 272 (1999) 173

[4] S.N. Dorogovtsev, J.F.F. Mendes, Phys. Rev. E 63 (2001) 025101

[5] D.H. Shi, Q.H. Chen, L.M. Liu, Phys. Rev. E 71 (2005) 036140

[6] G. Bianconi, A.-L. Barabási, Europhys. Lett. 54 (2001) 436.

[7] S.N. Dorogovtsev, J.F.F. Mendes, A.N. Samukhin, Phys. Rev. Lett. 85 (2000) 4633.

[8] J.L. Guo, C.P. Wang, Impuls. Dyn. Syst. Appl. 4 (2006) 970.

[9] J.L. Guo, Y.Q. Bai, Dyn. Contin. Discrete Impuls. Syst. Ser. B 13 (2006) 523.

[10] C.P. Zhu, T. Zhou, H.J. Yang, S.J. Xiong, Z.M. Gu, D.N. Shi, D.R. He, B.H. Wang, New J. Phys. 10 (2008) 023006.

[11] J.-G. Liu, Y.-Z. Dang, Z.-T. Wang, T. Zhou, Physica A 371 (2006) 861.

[12] Z. Liu, Y.-C. Lai, N. Ye, P. Dasgupta, Phys. Lett. A 303 (2002) 337.

[13] Q. Guo, T. Zhou, J.-G. Liu, W.-J. Bai, B.-H. Wang, M. Zhao, Physica A 371 (2006) 2006.

[14] X. Li, Y.Y. Jin, G.R. Chen, Physica A 328 (2003) 287.

[15] B.J. Kim, C.N. Yoon, S.K. Han, H. Jeong, Phys. Rev. E 65 (2002) 027103

[16] W.X. Wang, B.H. Wang, C.Y. Yin, Y.B. Xie, T. Zhou, Phys. Rev. E 73 (2006) 026111.

[17] X. Li, G.R. Chen, Physica A 328 (2003) 274.

[18] Z.Z. Zhang, L.L. Rong, B. Wang, S.G. Zhou, J.H. Guan, Physica A 380 (2007) 639.

[19] G.R. Chen, Z.P. Fan, X. Li, Modeling the complex Internet Topology, in: G. Vattay, L. Kocarev (Eds.), Complex Dynamics in Communication Networks, Springer-Verlag, Berlin, 2005.

[20] X. Wu, Z. Liu, Physica A 387 (2008) 623.

[21] M.E.J. Newman, M. Girvan, Phys. Rev. E 69 (2004) 026113.

[22] P.L. Krapivsky, S. Redner, F. Leyvraz, Phys. Rev. Lett. 85 (2000) 4629.

[23] P.L. Krapivsky, S. Redner, Phys. Rev. E 63 (2001) 066113.

[24] Z. Liu, Y.-C. Lai, N. Ye, Phys. Rev. E 66 (2002) 036112.

[25] T. Zhou, B.H. Wang, Y.D. Jin, D.R. He, P.P. Zhang, Y. He, B.B. Su, K. Chen, Z.Z. Zhang, J.G. Liu, Int. J. Mod. Phys. C 18 (2007) 297.

[26] D. Gross, C.M. Harris, Fundamentals of Queuing Theory, Wiley, New York, 1998.

27] E. Ravasz, A.-L. Barabási, Phys. Rev. E 67 (2003) 026112

[28] T. Zhou, G. Yan, B.H. Wang, Phys. Rev. E 71 (2005) 046141.

[29] P. Holme, B.J. Kim, Phys. Rev. E 65 (2002) 026107.

[30] T. Zhou, B.-H. Wang, P.-M. Hui, K.P. Chan, Physica A 367 (2006) 613. 